\title{
PREHISTORIC COLONIZATION OF NORTHEASTERN SIBERIA AND MIGRATION TO AMERICA: RADIOCARBON EVIDENCE ${ }^{1}$
}

\author{
YAROSLAV V. KUZMIN
}

Pacific Institute of Geography, Far Eastern Branch of the Russian Academy of Sciences Radio Street 7, Vladivostok 690041 Russia

\begin{abstract}
This review of radiocarbon dates from northeastern Siberian Paleolithic sites provides data that can be applied to establishing a chronology of human settlement, and that can provide a rough estimate of the timing of the initial peopling of the New World.
\end{abstract}

\section{INTRODUCTION}

Radiocarbon dating of ancient sites is one of the most useful and important aspects of chronometric age determination (Aitken 1990). This method is used extensively to establish the chronologies of both northeast Siberian and northwest North American Paleolithic cultures and migrations to the New World (see, e.g., Morlan 1987; Hoffecker, Powers and Goebel 1993; Whitley and Dorn 1993). Waters (1985) made one of the most appropriate critical overviews of ${ }^{14} \mathrm{C}$ dating for the earliest (pre-Clovis) sites in the Americas. The aim of this review is to present the ${ }^{14} \mathrm{C}$ dates of northeast Siberia, together with a brief discussion of their relevance to an important scientific problem-the peopling of the New World. Because the majority of sources of Siberian ${ }^{14} \mathrm{C}$ dates are in Russian, it is useful to compile an English-language review for the international scientific community.

\section{METHODS}

Table 1 lists the ${ }^{14} \mathrm{C}$ dates from Paleolithic sites of northeast Siberia, from Lake Baikal to Chukotka (Figs. 1-3). The list includes dates from published sources through Autumn 1993, but includes no references. Besides the dates, Table 1 includes such information as sample material, context and depth below surface. Dates are grouped into geographical regions of northeastern Siberia.

\section{Discussion}

\section{Early Upper Paleolithic (39,000-24,000 BP)}

Ancient sites that are associated with the Early Upper Paleolithic have not been ${ }^{14} \mathrm{C}$ dated, except for the Filimoshki site (Table 1). Up to the present, Early Paleolithic and Mousterian sites of northeastern Siberia are unknown in well-defined geological contexts (Yi and Clark 1983; Kuzmin 1992a; Kuzmin and Krivonogov 1994). The earliest Upper Paleolithic sites of the area are Makarovo 4, Arta 2 (Layer 4), Tolbaga (Layer 4), Varvarina Gora (Layer 2), Ust-Kova (Layer 7) and Geographical Society Cave (Fig. 1). Dates of these sites range from 39,000 to 27,200 BP.

Kirillov and Kasparov (1990) assumed that Layer 4 of the Arta 2 site is associated with the cultural stage preceding the Upper Paleolithic. Mochanov (1977) used ${ }^{14} \mathrm{C}$ dates from the Ust-Mil 2 (Layers $\mathrm{B}, \mathrm{C}$ ) and Ikhine 2 (Layers $2 \mathrm{~b}, \mathrm{c}, \mathrm{d}$ ) sites to suggest that the beginning of the Dyutkai complex lies between 30,000 and 35,000 BP. Some archaeologists, for example, Abramova (1979) do not accept this viewpoint, and propose that the ages of these sites are between 15,000 and 20,000 BP. One of

\footnotetext{
${ }^{1}$ Editors' note: This paper was scheduled for presentation at the Archaeology Workshop of the 15th International Radiocarbon Conference, 14 August 1994, Glasgow, Scotland, but was not delivered because of the author's absence.
} 
the earliest of Mochanov's key sites - Ezhantsy - was dated, at the end of the 1970s, to 17,150 \pm 345 BP: IM-459. In my opinion, the most probable age of the earliest Yakutian sites is ca. 24,000 $26,000 \mathrm{BP}$. Thus, the general boundary between the early and late stages of the Upper Paleolithic in northeastern Siberia and may be fixed to $c a .29,000-24,000 \mathrm{BP}$.

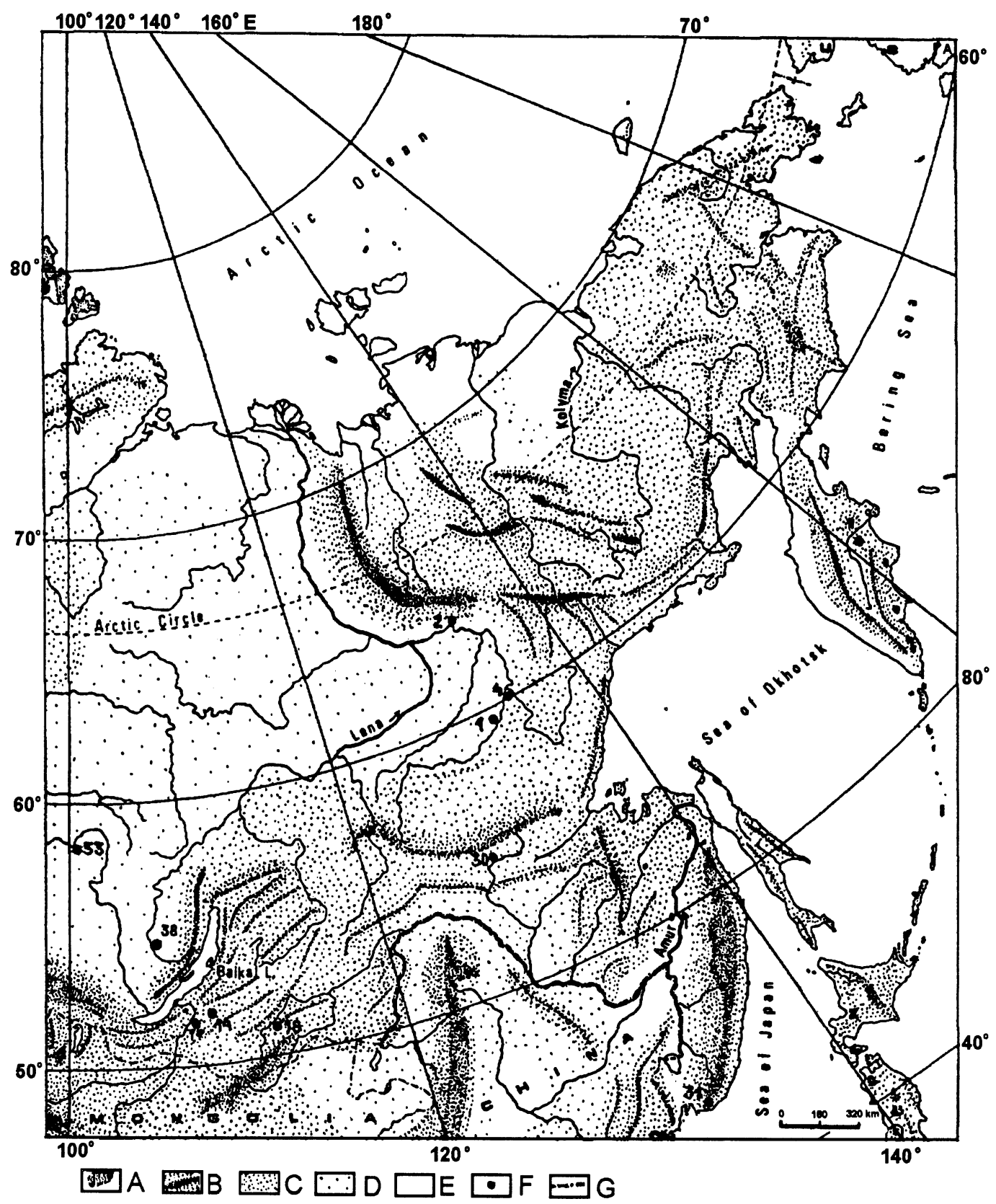

Fig. 1. The early Upper Paleolithic sites in northeast Siberia (numbers correspond to those in Table 1). A - modern glaciers; B - mountain ridges; C - high plains; D - low plains; E-lowlands; F - Paleolithic sites; G - state boundaries. 


\section{Late Upper Paleolithic (24,000-10,000 BP)}

Many ancient sites in northeastern Siberia relate to the Late Upper Paleolithic, occurring practically all over the study region, except for the Kolyma River basin and Chukotka (Fig. 2). The earliest sites of this cultural stage are Military Hospital 2 and Makarovo 3, containing redeposited material and dating from 31,200-29,700 BP. Most of the dates fall within the period, 24,000-10,000 BP, whereas, in Yakutia, the Ust-Timpton site, Layer 5a is as late as $9400 \mathrm{BP}$.

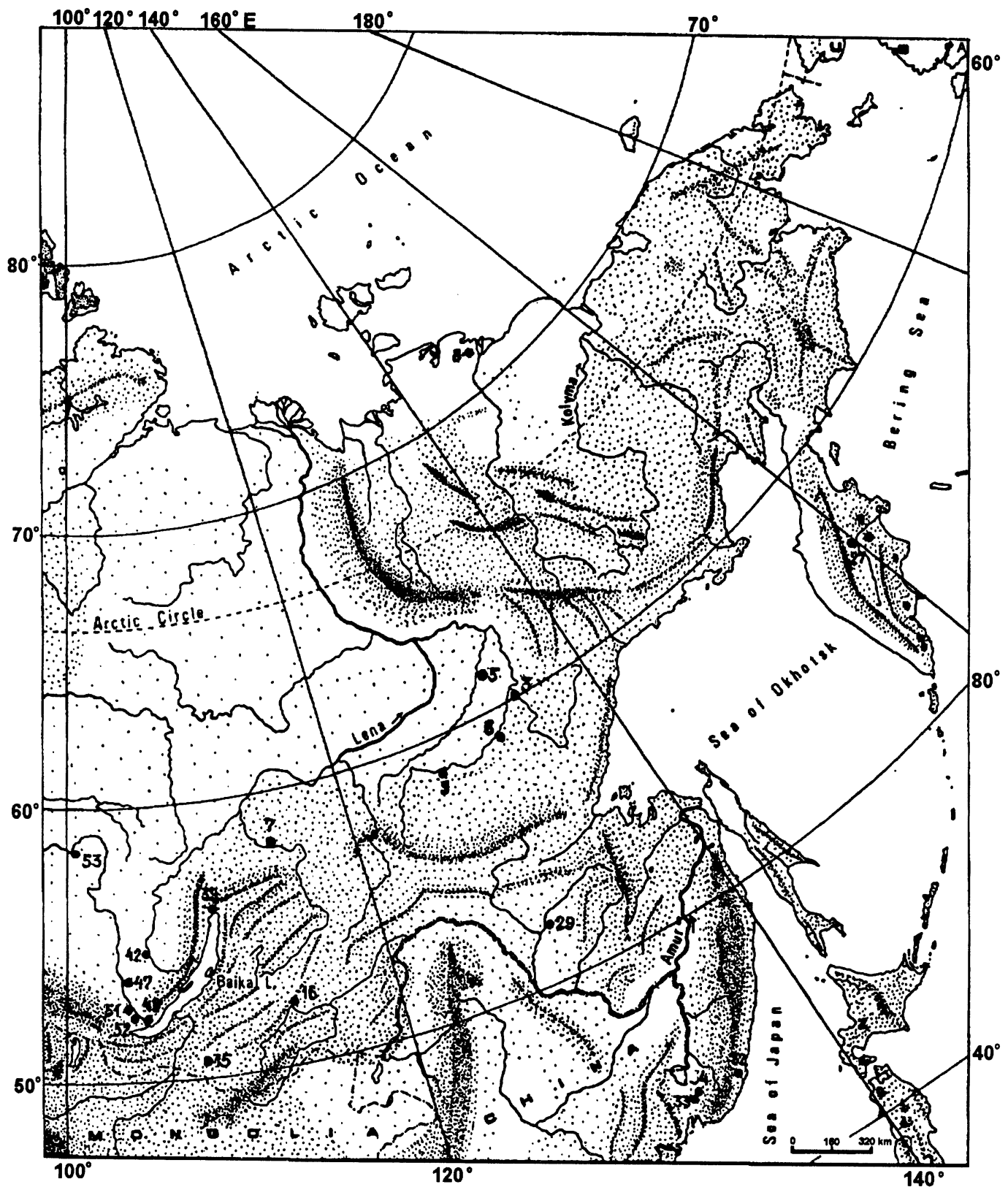

Fig. 2. Late Upper Paleolithic sites in northeast Siberia (numbers correspond to those in Table 1). For key to symbols, see Fig. 1. 


\section{Late to Final Paleolithic Transition (15,200-12,400 BP)}

The earliest sites associated with the transition from Late to Final Paleolithic are Makarovo 2, Ust-Belaya (Layer 14), Kurla 3 (Layer 1) and Bolshoi Yakor (Layer 7), which range from 15,200 to $12,400 \mathrm{BP}$.

\section{Final Upper Paleolithic (Mesolithic) (10,000-6000 BP)}

Sites of this cultural stage occupy all of northeastern Siberia (Fig. 3).

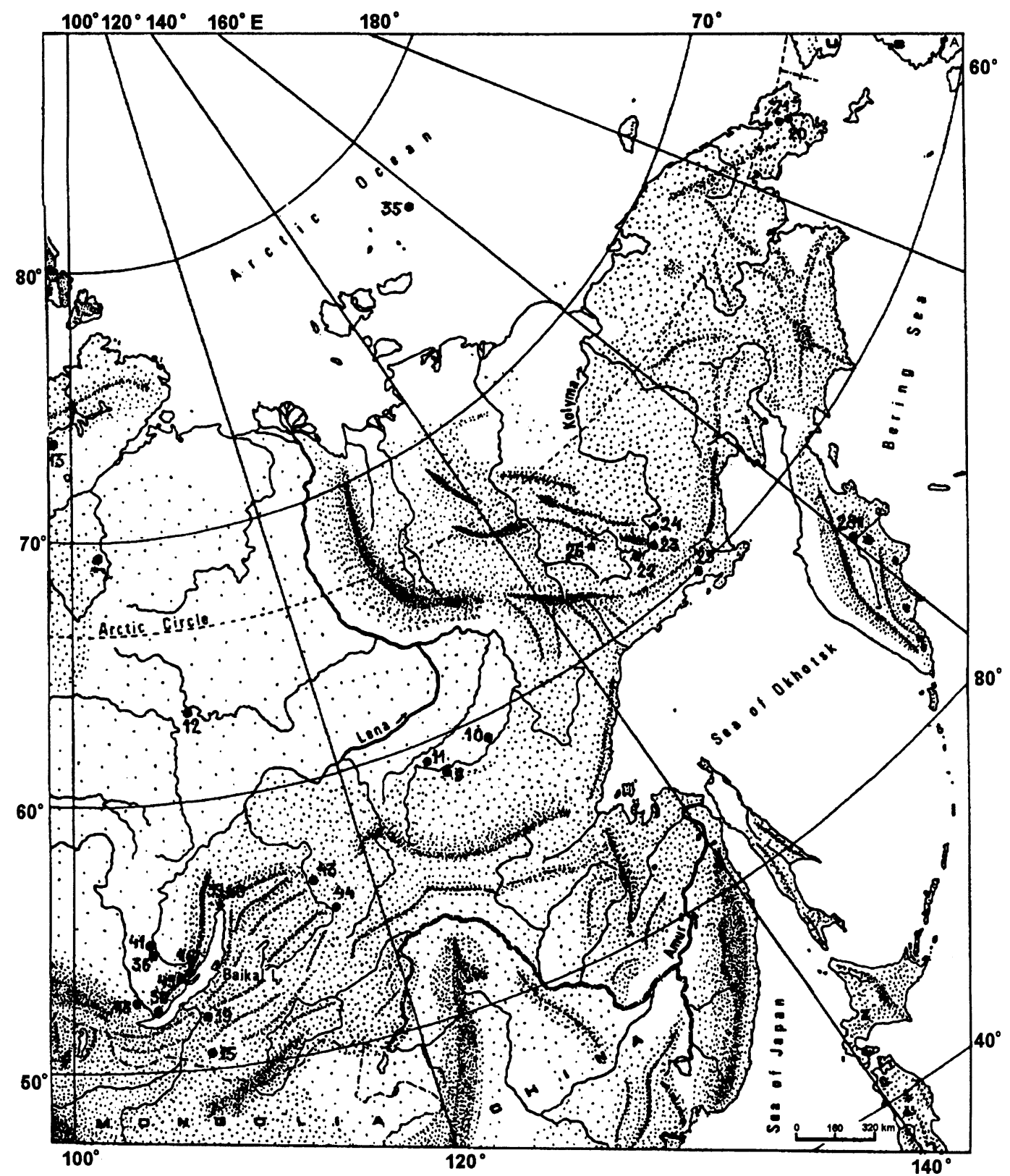

Fig. 3. Final Upper Paleolithic sites in northeast Siberia. For key to symbols, see Fig. 1. 


\section{Paleolithic to Neolithic Transition (12,960-5900 BP)}

Dating not later than 5900 BP, the younger sites are located in Yakutia, Taimyir Peninsula, and in the High Arctic, are Belkachi, Sumnagin, Tagenar 6 and Zhokhov. The Paleolithic/Neolithic transition in northeastern Siberia is non-synchronous. Taking into account that pottery-making is the most distinctive innovation of the Neolithic, the earliest pottery of the area comes from the Lower Amur River basin - the Gasya site, dating to $12,960 \pm 120 \mathrm{BP}$ : LE-1781, and from Trans-baikal, dating to $c a .10,000 \mathrm{BP}$. At ca. $8000 \mathrm{BP}$, pottery appeared for the first time over most of the Russian Far East (Kuzmin 1992a, b). In Yakutia, the Kolyma River basin and Chukotka, the earliest pottery dated to younger than $6000 \mathrm{BP}$.

TABLE 1. Radiocarbon Dates from the Paleolithic and Mesolithic Sites in Northeastern Siberia

\begin{tabular}{|c|c|c|c|c|}
\hline Site no., name* & $\begin{array}{c}{ }^{14} \mathrm{C} \text { date } \\
\text { (yr BP) }\end{array}$ & Lab no. & Sample material & Context, depth \\
\hline \multicolumn{5}{|l|}{ Yakutia } \\
\hline \multirow[t]{6}{*}{1 Ust-Mil 2} & $12,200 \pm 170$ & LE-953 & Wood & Layer A, $1.50 \mathrm{~m}$ \\
\hline & $23,500 \pm 500$ & LE-999 & Wood & Layer B, $1.80 \mathrm{~m}$ \\
\hline & $33,000 \pm 500$ & LE-1000 & Wood & Layer C, $2.25 \mathrm{~m}$ \\
\hline & $30,000 \pm 500$ & LE-1001 & Wood & Layer C, $2.40 \mathrm{~m}$ \\
\hline & $35,400 \pm 600$ & LE-954 & Wood & Layer C, $2.50 \mathrm{~m}$ \\
\hline & $35,600 \pm 900$ & LE-955 & Wood & Below artifacts, $4.0 \mathrm{~m}$ \\
\hline \multirow[t]{10}{*}{2 Ikhine 2} & $24,330 \pm 200$ & LE-1131 & Wood & Layer IIb, $0.95 \mathrm{~m}$ \\
\hline & $24,500 \pm 480$ & IM-203 & Wood & Layer IIb, $0.90 \mathrm{~m}$ \\
\hline & $24,600 \pm 380$ & IM-155 & Wood & Layer IIc, $1.40 \mathrm{~m}$ \\
\hline & $27,400 \pm 800$ & IM-205 & Wood & Layer IIb, $0.90 \mathrm{~m}$ \\
\hline & $30,200 \pm 300$ & GIN-1019 & Wood & Layer IIb, $0.95 \mathrm{~m}$ \\
\hline & $26,600 \pm 900$ & IM-201 & Wood & Layer IIc, $1.20 \mathrm{~m}$ \\
\hline & $31,200 \pm 500$ & GIN-1020 & Wood & Layer IIc, $1.20 \mathrm{~m}$ \\
\hline & $26,030 \pm 200$ & IM-239 & Bone & Rhinoceros, $1.30 \mathrm{~m}$ \\
\hline & $26,500 \pm 540$ & IM-202 & Wood & Layer IIc, $1.30 \mathrm{~m}$ \\
\hline & $27,800 \pm 500$ & IM-206 & Wood & Layer IId, $1.60 \mathrm{~m}$ \\
\hline 3 Verkhne-Troitskaya & $18,300 \pm 180$ & LE-905 & Wood & $5.30 \mathrm{~m}$ \\
\hline 4 Ezhantsy & $17,150 \pm 345$ & IM-459 & Bone & Alluvium, $0.60-1.0 \mathrm{~m}$ \\
\hline 5 Khaergas & $16,000 \pm 300$ & IM-887 & Bone & Layer 6 \\
\hline \multirow[t]{7}{*}{6 Duyktai Cave } & $12,100 \pm 120$ & LE-907 & Wood & Layer VIIa, $1.80 \mathrm{~m}$ \\
\hline & $12,520 \pm 260$ & IM-462 & Wood & $\begin{array}{l}\text { Layer VIIb mammoth, } \\
2.7 \mathrm{~m}\end{array}$ \\
\hline & $12,690 \pm 120$ & LE-860 & Charcoal & Layer VIIb, $2.60 \mathrm{~m}$ \\
\hline & $13,070 \pm 90$ & LE-784 & Charcoal & Layer VIIb, $2.30 \mathrm{~m}$ \\
\hline & $13,110 \pm 90$ & LE-908 & Wood & Layer VIIc, $3.50 \mathrm{~m}$ \\
\hline & $13,200 \pm 250$ & GIN-405 & Charcoal & Layer VIIa, $1.60 \mathrm{~m}$ \\
\hline & $14,000 \pm 100$ & GIN-404 & Charcoal & Layer VIIb, $2.30 \mathrm{~m}$ \\
\hline \multirow[t]{3}{*}{7 Avdeikha } & $9200 \pm 390$ & IM-471 & ? & $0.90 \mathrm{~m}$ \\
\hline & $12,900 \pm 300$ & GIN-1022 & Charcoal & Layer C, $0.90 \mathrm{~m}$ \\
\hline & $15,200 \pm 300$ & IM-236 & Charcoal & Layer C, $0.80-1.20 \mathrm{~m}$ \\
\hline \multirow[t]{5}{*}{8 Berelekh } & $10,600 \pm 90$ & LE-998 & Wood & $2.53 \mathrm{~m}$ \\
\hline & $11,830 \pm 110$ & LU-147 & Wood & Above artifacts, $1.6 \mathrm{~m}$ \\
\hline & $12,240 \pm 160$ & LU-149 & Ivory & $2.55 \mathrm{~m}$ \\
\hline & $12,930 \pm 80$ & GIN-1021 & Wood & $2.30 \mathrm{~m}$ \\
\hline & $13,420 \pm 200$ & IM-152 & Wood & $2.53 \mathrm{~m}$ \\
\hline \multirow[t]{4}{*}{9 Ust-Timpton } & $6380 \pm 80$ & LE-894 & Charcoal & Layer IIIb, $1.20 \mathrm{~m}$ \\
\hline & $6570 \pm 100$ & LE-910 & Charcoal & Layer IIIb, $1.25 \mathrm{~m}$ \\
\hline & $7000 \pm 90$ & LE-895 & Charcoal & Layer IVa, $1.50 \mathrm{~m}$ \\
\hline & $8900 \pm 200$ & IM-456 & Charcoal & Layer Va, $2.00 \mathrm{~m}$ \\
\hline
\end{tabular}


TABLE 1. (Continued)

\begin{tabular}{|c|c|c|c|c|}
\hline Site no., name* & $\begin{array}{c}{ }^{14} \mathrm{C} \text { date } \\
\text { (yr BP) }\end{array}$ & Lab no. & Sample material & Context, depth \\
\hline \multirow{28}{*}{10 Belkachi } & $9000 \pm 110$ & LE-832 & Charcoal & Layer IVb, $1.75 \mathrm{~m}$ \\
\hline & $9400 \pm 90$ & LE-896 & Charcoal & Layer Va, $2.00 \mathrm{~m}$ \\
\hline & $9450 \pm 300$ & IM-455 & Charcoal & Layer $\mathrm{Vb}, 2.05 \mathrm{~m}$ \\
\hline & $10,130 \pm 100$ & LE-897 & Wood & Layer VIb, $2.25 \mathrm{~m}$ \\
\hline & $10,300 \pm 50$ & LE-920 & Charcoal & Between L. V-VI, $2.15 \mathrm{~m}$ \\
\hline & $10,340 \pm 140$ & LE-862 & Wood & Layer VIa, $2.20 \mathrm{~m}$ \\
\hline & $10,650 \pm 80$ & LE-898 & Wood & Layer VIb, $2.30 \mathrm{~m}$ \\
\hline & $10,740 \pm 100$ & LE-861 & Charcoal & Layer VIb, $2.05 \mathrm{~m}$ \\
\hline & $11,150 \pm 150$ & IM-454 & Charcoal & Layer V (?) \\
\hline & $11,800 \pm 200$ & IM-453 & Charcoal & Layer VIII, $2.50 \mathrm{~m}$ \\
\hline & $5900 \pm 70$ & LE-678 & Charcoal & Layer $8,1.80 \mathrm{~m}$ \\
\hline & $6250 \pm 60$ & LE-697 & Charcoal & Layer 9, $2.00 \mathrm{~m}$ \\
\hline & $6720 \pm 50$ & LE-650 & Charcoal & Layer $10,3.00 \mathrm{~m}$ \\
\hline & $6750 \pm 70$ & LE-698 & Charcoal & Layer $10,3.10 \mathrm{~m}$ \\
\hline & $7430 \pm 60$ & LE-741 & Wood & Layer $12,2.90 \mathrm{~m}$ \\
\hline & $7830 \pm 150$ & LE-742 & Wood & Layer $13,3.30 \mathrm{~m}$ \\
\hline & $7920 \pm 60$ & LE-743 & Wood & Layer $14,3.70 \mathrm{~m}$ \\
\hline & $8060 \pm 70$ & LE-746 & Charcoal & Layer $17,5.10 \mathrm{~m}$ \\
\hline & $8110 \pm 80$ & LE-744 & Wood & Layer $15,4.60 \mathrm{~m}$ \\
\hline & $8260 \pm 80$ & LE-745 & Wood & Layer $17,4.90 \mathrm{~m}$ \\
\hline & $8290 \pm 80$ & LE-760 & Wood & Layer $19,5.50 \mathrm{~m}$ \\
\hline & $8360 \pm 80$ & LE-747 & Wood & Layer $18,5.30 \mathrm{~m}$ \\
\hline & $8370 \pm 80$ & LE-761 & Charcoal & Layer $20,5.80 \mathrm{~m}$ \\
\hline & $8440 \pm 80$ & LE-801 & Wood & Layer $21,5.90 \mathrm{~m}$ \\
\hline & $8500 \pm 160$ & LE-740 & Charcoal & Close to L. $20,5.80 \mathrm{~m}$ \\
\hline & $8520 \pm 80$ & LE-762 & Charcoal & Layer $22,6.10 \mathrm{~m}$ \\
\hline & $9045 \pm 210$ & IM-243 & Wood & Layer $23,6.30 \mathrm{~m}$ \\
\hline & $9180 \pm 80$ & LE-763 & Wood & Layer $23,6.30 \mathrm{~m}$ \\
\hline \multirow[t]{6}{*}{11 Sumnagin } & $5960 \pm 60$ & LE-795 & $?$ & Layer $20,2.70 \mathrm{~m}$ \\
\hline & $6100 \pm 50$ & GIN-296 & Wood & Layer $36,4.45 \mathrm{~m}$ \\
\hline & $6200 \pm 60$ & LE-798 & $?$ & Layer $36,4.45 \mathrm{~m}$ \\
\hline & $6280 \pm 60$ & LE-797 & $?$ & Layer $33,4.20 \mathrm{~m}$ \\
\hline & $6360 \pm 60$ & LE-796 & $?$ & Layer $24,3.10 \mathrm{~m}$ \\
\hline & $6900 \pm 50$ & GIN-295 & Wood & Layer $20,2.70 \mathrm{~m}$ \\
\hline \multirow[t]{6}{*}{12 Ust-Chirkuo } & $7200 \pm 180$ & IM-475 & Wood & Layer $5,1.20 \mathrm{~m}$ \\
\hline & $7600 \pm 80$ & LE-996 & $?$ & Layer $6,0.80 \mathrm{~m}$ \\
\hline & $7650 \pm 170$ & IM-481 & Charcoal & Layer $8,1.40 \mathrm{~m}$ \\
\hline & $8350 \pm 150$ & IM-476 & Wood & Layer $12,1.80 \mathrm{~m}$ \\
\hline & $8740 \pm 100$ & IM-373 & $?$ & Layer $10,1.60 \mathrm{~m}$ \\
\hline & $8750 \pm 200$ & IM-479 & Charcoal & Layer $10,1.60 \mathrm{~m}$ \\
\hline 13 Tagenar 6 & $6030 \pm 100$ & LE-884 & $?$ & \\
\hline \multicolumn{5}{|l|}{ Transbaikalian } \\
\hline \multirow[t]{3}{*}{14 Tolbaga } & $15,100 \pm 520$ & SOAN-810 & Bone & Layer 3 \\
\hline & $27,210 \pm 300$ & SOAN-1523 & Bone & Layer 4 \\
\hline & $34,860 \pm 2100$ & SOAN-1522 & Bone & Rhinoceros, Layer 4 \\
\hline \multirow[t]{8}{*}{15 Studenoye } & $10,755 \pm 140$ & SOAN-1653 & Coal & Layer $13 / 1$ \\
\hline & $10,975 \pm 135$ & SOAN-1654 & Charcoal & Layer 14 \\
\hline & $11,395 \pm 100$ & SOAN-1655 & Charcoal & Layer 14 \\
\hline & $11,340 \pm 180$ & GIN-2931a & Charcoal & Layer 15 \\
\hline & $11,660 \pm 400$ & GIN-2930 & Charcoal & Layer 15 \\
\hline & $11,340 \pm 200$ & GIN-2932 & Charcoal & Layer 16 \\
\hline & $11,630 \pm 50$ & SOAN-1656 & Charcoal & Layer 16 \\
\hline & $12,130 \pm 150$ & GIN-2934a & Charcoal & Layer 17 \\
\hline
\end{tabular}


TABLE 1. (Continued)

\begin{tabular}{|c|c|c|c|c|}
\hline Site no., name* & $\begin{array}{c}{ }^{14} \mathrm{C} \text { date } \\
\text { (yr BP) }\end{array}$ & Lab no. & Sample material & Context, depth \\
\hline & $12,140 \pm 150$ & GIN-2934 & Charcoal & Layer 17 \\
\hline & $12,110 \pm 150$ & GIN-2935 & Charcoal & Layer 18 \\
\hline & $12,800 \pm 400$ & GIN-2937 & Charcoal & Layer 18 \\
\hline \multirow[t]{2}{*}{16 Sokhatino 4} & $11,900 \pm 130$ & SOAN-841 & Bone & \\
\hline & $26,110 \pm 200$ & SOAN-1138 & Charcoal & \\
\hline \multirow[t]{4}{*}{17 Varvarina Gora } & $17,035 \pm 400$ & SOAN-3053 & $?$ & Layer 1 \\
\hline & $29,895 \pm 1790$ & SOAN-3054 & $?$ & Layer 2 \\
\hline & $30,600 \pm 500$ & SOAN-850 & Bone & Layer 2 \\
\hline & $34,900 \pm 780$ & SOAN-1524 & Bone & Layer 2 \\
\hline \multirow[t]{2}{*}{18 Arta 2} & $23,200 \pm 2000$ & LE-2966 & Charcoal & Layer 3 \\
\hline & $37,360 \pm 2000$ & LE-2967 & Charcoal & Mousterian(?), Layer 4 \\
\hline 19 Oshurkovo & $10,900 \pm 500$ & GIN-302 & Charcoal & Layer 3 \\
\hline \multicolumn{5}{|l|}{ Chukotka } \\
\hline 20 Chelkun 4 & $8150 \pm 450$ & MAG-719 & Charcoal & \\
\hline 21 Ananaiveem & $8410 \pm 80$ & LE-2791 & Charcoal & \\
\hline \multicolumn{5}{|l|}{ Kolyma River Basin } \\
\hline \multirow[t]{6}{*}{22 Siberdik } & $7865 \pm 310$ & MAG-184 & Charcoal & Layer 3, $0.90 \mathrm{~m}$ \\
\hline & $8020 \pm 80$ & KRIL-250 & Charcoal & Layer $3,0.80-0.90 \mathrm{~m}$ \\
\hline & $8130 \pm 100$ & MAG-606 & $?$ & Layer $3,0.80 \mathrm{~m}$ \\
\hline & $8480 \pm 200$ & KRIL-249 & Charcoal & Layer $3,0.80-0.90 \mathrm{~m}$ \\
\hline & $9700 \pm 500$ & MAG-1019 & $?$ & Layer $3,1.00 \mathrm{~m}$ \\
\hline & $13,225 \pm 230$ & MAG-916 & $?$ & Layer 3 \\
\hline \multirow[t]{6}{*}{23 Kongo } & $8080 \pm 500$ & MAG-406 & $?$ & Layer 1 \\
\hline & $8600 \pm 220$ & MAG-196 & Charcoal & Layer $1,1.60 \mathrm{~m}$ \\
\hline & $8700 \pm 400$ & MAG-595 & $?$ & Layer 3, (?) \\
\hline & $8850 \pm 500$ & KRIL-315 & Charcoal & Layer $2,1.00 \mathrm{~m}$ \\
\hline & $9020 \pm 510$ & KRIL-313 & Charcoal & Layer $2,1.00 \mathrm{~m}$ \\
\hline & $9470 \pm 530$ & KRIL-314 & Charcoal & Layer $2,1.20 \mathrm{~m}$ \\
\hline 24 Maltan & $7490 \pm 70$ & MAG-183 & Charcoal & Layer $2,0.40 \mathrm{~m}$ \\
\hline 25 Uptar & $8260 \pm 330$ & MAG-1262 & Charcoal & $0.10-0.35 \mathrm{~m}$ \\
\hline 26 Zima & $7070 \pm 60$ & MAG-1260 & Charcoal & \\
\hline \multicolumn{5}{|l|}{ Kamchatka } \\
\hline \multirow[t]{7}{*}{27 Ushki 1} & $9750 \pm 100$ & MAG-637 & Charcoal & Layer 7 \\
\hline & $10,360 \pm 220$ & MAG-401 & Charcoal & Layer 6 \\
\hline & $10,360 \pm 350$ & MO-345 & Charcoal & Layer $6,1.70 \mathrm{~m}$ \\
\hline & $10,760 \pm 110$ & MAG-219 & Charcoal & Layer $6,1.80 \mathrm{~m}$ \\
\hline & $13,600 \pm 250$ & GIN-167 & Charcoal & Layer $7,2.20 \mathrm{~m}$ \\
\hline & $14,300 \pm 200$ & GIN-168 & Charcoal & Layer 7 \\
\hline & $21,000 \pm 100$ & GIN-186 & Charcoal & Layer 6 (?) \\
\hline 28 Ushki 5 & $8790 \pm 150$ & MAG-215 & Charcoal & Layer 6 \\
\hline \multicolumn{5}{|l|}{ Russian Far East } \\
\hline 29 Ust-Ulma & $19,350 \pm 65$ & SOAN-2619 & Charcoal hearth & \\
\hline 30 Filimoshki & $20,350 \pm 850$ & SOAN-825 & Peat & Redeposited \\
\hline $\begin{array}{l}31 \text { Geographical } \\
\text { Society Cave }\end{array}$ & $32,570 \pm 1510$ & IGAN-341† & Bone & $\begin{array}{l}\text { Horse, mammoth, } \\
0.60-0.80 \mathrm{~m}\end{array}$ \\
\hline \multirow[t]{2}{*}{32 Suvorovo 4} & $15,105 \pm 140$ & AA-9463† & Charcoal & $0.25-0.30 \mathrm{~m}$ \\
\hline & $15,300 \pm 140$ & $\mathrm{Ki}-3502 \dagger$ & Charcoal & $0.25-0.30 \mathrm{~m}$ \\
\hline 33 Gorbatka 3 & $13,500 \pm 200$ & SOAN-1922† & Humic acids & Below artifacts \\
\hline 34 Ilistaya 1 & $7840 \pm 60$ & $\mathrm{Ki}-3163 \dagger$ & Charcoal & \\
\hline \multicolumn{5}{|l|}{ High Arctic } \\
\hline 35 Zhokhov Island & $7450 \pm 200$ & LE-4534 & Wood & \\
\hline
\end{tabular}


TABLE 1. (Continued)

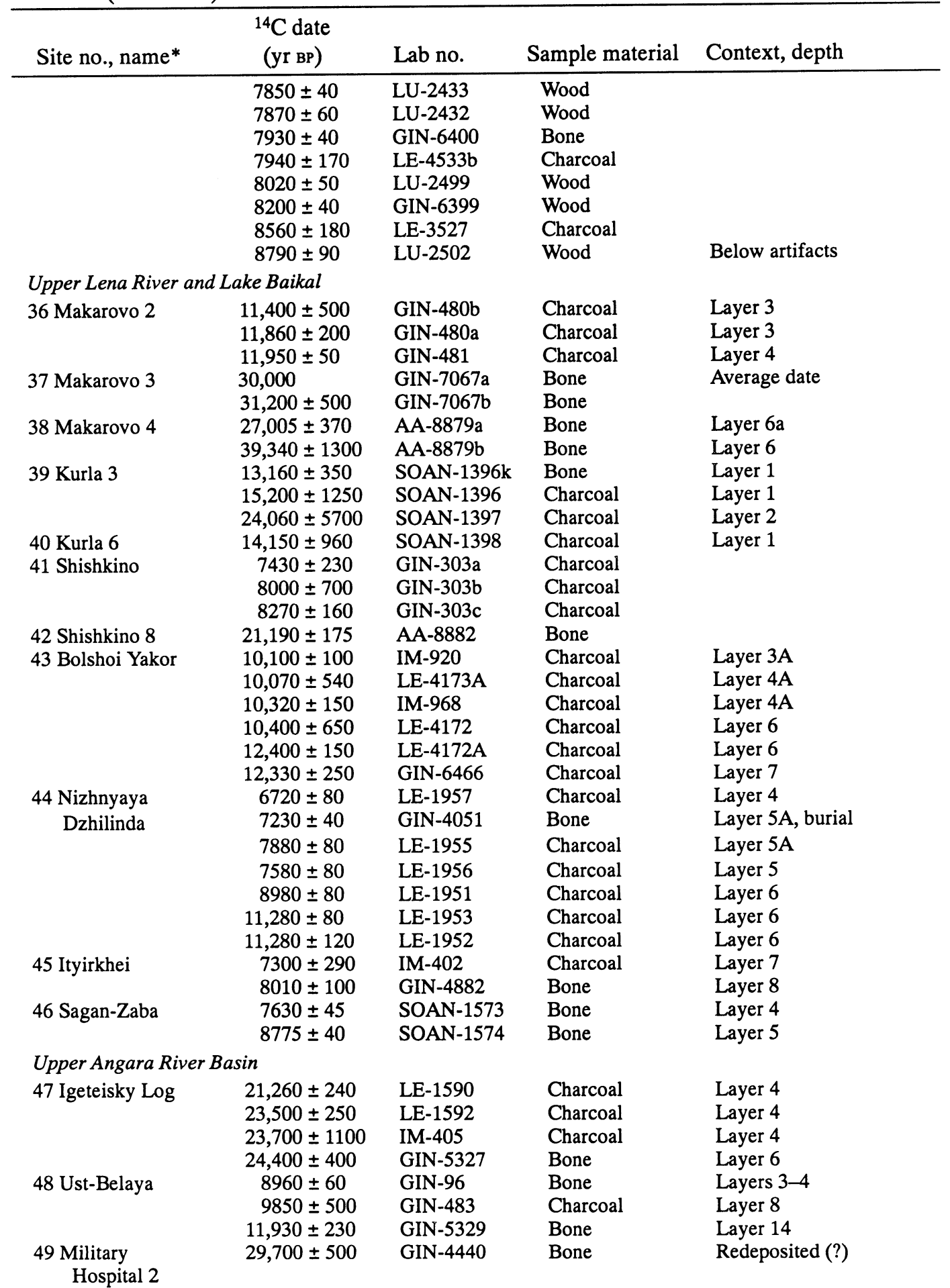


TABLE 1. (Continued)

\begin{tabular}{lclll}
\hline Site no., name* & $\begin{array}{c}14 \text { C date } \\
(\mathrm{yr} \text { BP })\end{array}$ & Lab no. & Sample material & Context, depth \\
\hline $\begin{array}{c}50 \text { Verkholenskaya } \\
\text { Gora } 1\end{array}$ & $12,570 \pm 180$ & MO-441 & Charcoal & Layer 3 \\
51 Buret & & & & \\
& $21,190 \pm 100$ & $?$ & Bone & \\
52 Malta & $14,750 \pm 120$ & GIN-97 & Bone & Badai horizon \\
& $20,700 \pm 150$ & GIN-7709 & Bone & \\
53 Ust-Kova & $21,000 \pm 140$ & GIN-7706 & Bone & \\
& $14,220 \pm 100$ & LE-1372 & Charcoal & Layer 4 \\
& $23,920 \pm 310$ & KRIL-381 & Charcoal & Layer 5 \\
& $28,050 \pm 670$ & SOAN-1875 & Charcoal & Layer 7 \\
& $30,100 \pm 150$ & GIN-1741 & Charcoal & Layer 7 \\
& $>32,865$ & SOAN-1874 & Charcoal & Layer 7 \\
\hline
\end{tabular}

*Numbers correspond to numbers in Figs. 1-3.

†Also reported in Kuzmin et al. (1994), which precedes this article.

\section{CONCLUSION}

From the available data, it is possible to draw a preliminary conclusion that ancient people occupied western Beringia (the Indigirka River basin and Kamchatka) ca. 12,000-14,000 BP, at which time they could have migrated across the Bering Land Bridge. The data presented here form the foundation for a proposed Radiocarbon Database of Paleolithic sites in Siberia and the Russian Far East. Because of the rapid accumulation of new dates, it is necessary to plan for a sustained supplement to the database. For a detailed discussion of the general database, see Kra (1988).

\section{ACKNOWLEDGMENTS}

I am grateful to the following persons who helped me in the preparation of the manuscript and in the discussion of the data: M. P. Aksenov; O. I. Goryunova; E. M. Ineshin; G. I. Medvedev; L. D. Sulerzhitsky; and V. N. Vetrov. My thanks also go to M. R. Waters for the idea to compile this review. I am especially grateful to Dr. A. J. T. Jull and Profs. Robert E. Ackerman and Don E. Dumond for correcting the language and grammar of the draft. Research for this publication was supported by Grant \#122-0645 A from the International Research and Exchanges Board, with funds provided by the U.S. Department of State, Title VIII. Neither of these organizations is responsible for the views expressed in the manuscript.

\section{REFERENCES}

Abramova, Z. A. 1979 On the dating of the Paleolithic of Aldan River. Soviet Archaeology 4: 5-14 (in Russian).

Aitken, M. J. 1990 Science-Based Dating in Archaeology. London, Longman: 294 p.

Hoffecker, J. F., Powers, W. R. and Goebel, T. 1993 The colonization of Beringia and the peopling of the New World. Science 259 (1): 46-53.

Kirillov, I. I. and Kasparov, A. K. 1990 Archaeology of the Transbaikalian: Problems and perspectives. In Chronostratigraphy of the Paleolithic of Northern, Central and East Asia and America. Proceedings of the International Symposium. Novosibirsk, Institute of History, Philology and Philosophy, Siberian
Branch of the USSR Academy of Sciences: 194-198 (in Russian).

Kra, R. S. 1988 Updating the past: The establishment of the International Radiocarbon Data Base. American Antiquity 53(1): 118-125.

Kuzmin, Y. V. 1992a Paleoecology of the Paleolithic of the Russian Far East. Sunsa Munhwa (Bulletin of the Institute of Prehistory) Chungbuk National University, Korea 1: 143-159.

1992b Paleogeography and chronology of ancient cultures of the Stone Age in Primorye. Russian Geology and Geophysics 33(6): 120-124. 
Kuzmin, Y. V. and Krivonogov, S. K. 1994 The Diring Paleolithic site, eastern Siberia: Review of geoarchaeological studies. Geoarchaeology 9(4): 287-300.

Mochanov, Y. A. 1977 The Earliest Stages of Human Occupation of Northeastern Asia. Novosibirsk, Nauka: 264 p.

Morlan, R. E. 1987 The Pleistocene archaeology of Beringia. In Nitecki, M. H. and Nitecki, D. V., eds., The Evolution of Human Hunting. New York, Plenum Press: 267-307.

Waters, M. R. 1985 Early Man in the New World: An evaluation of the radiocarbon dated Pre-Clovis sites in the Americas. In Mead, J. I. and Meltzer, D. J., eds., Environments and Extinctions: Man in Late Glacial North America. Orono, Maine, Center for the Study of Early Man: 125-143.

Whitley, D. S. and Dorn, R. I. 1993 New perspectives on the Clovis vs. Pre-Clovis controversy. American Antiquity 58 (4): 626-647.

Yi, S. and Clark, G. A. 1983 Observations on the Lower Paleolithic of Northeast Asia. Current Anthropology 24(2): 181-202. 